\title{
Heterogeneity Factor Analysis of Studies Evaluating the Effect of Tai Chi on Improving the Overall Quality of Life in Patients with Breast Cancer
}

\author{
Yuanqing Pan ${ }^{1, ~ *, ~ Y a n x u ~ Y a n g ~}{ }^{2}$, Yi Dong ${ }^{3}$, Nan $\mathrm{Li}^{4}$, Haiqian Liang ${ }^{5}$, Qi Cui \\ ${ }^{1}$ Department of Medical Psychology, Department of Public Course, Tianjin Medical College, Tianjin, China \\ ${ }^{2}$ Department of Public Health, Tianjin Medical College, Tianjin, China \\ ${ }^{3}$ Department of Pharmacy, Tianjin Medical College, Tianjin, China \\ ${ }^{4}$ Nursing Department of Neurosurgery, Tianjin Pingjin Hospital, Tianjin, China \\ ${ }^{5}$ Department of Neurosurgery, Tianjin Pingjin Hospital, Tianjin, China \\ ${ }^{6}$ The Department of Clinical Pathway and Quality Control the Frist Affiliated Hospital of Lanzhou University, Lanzhou, China \\ Email address: \\ panyuanq@126.com(Yuanqing Pan),lianghaiqiang@163.com (Haiqian Liang),zxsd411@126.com(Yi Dong),gaib@163.com(Nan Li), \\ yyx.wyj@163.com(Yanxu Yang),cuiqi_cs@126.com(Qi Cui) \\ ${ }^{*}$ Corresponding author
}

\section{To cite this article:}

Yuanqing Pan, Yanxu Yang, Yi Dong, Nan Li, Haiqian Liang, Qi Cui. Heterogeneity Factor Analysis of Studies Evaluating the Effect of Tai Chi on Improving the Overall Quality of Life in Patients with Breast Cancer. Clinical Medicine Research. Vol. 7, No. 3, 2018 , pp. 57-66.

doi: $10.11648 /$ j.cmr.20180703.12

Received: May 12, 2018; Accepted: June 4, 2018; Published: June 15, 2018

\begin{abstract}
Background: In the case of large heterogeneity, the data combination of randomized controlled trials will lead to the decrease of the reliability of systematic review and meta analysis, so we conducted the present study. Objectives: To explore sources of heterogeneity in studies on Tai Chi Chuan (TCC) as a complementary and alternative method for managing the overall quality of life of postoperative breast cancer patients. Methods: Randomized controlled trials were searched for in PubMed, EMBASE, Web of Science and the Cochrane Library through August 2017. The Cochrane Handbook 5.2 standards and Stata software version 10.0 were adopted for evaluating the quality of the included studies and the data was analyzed on overall quality of life of TCC. A separate univariate meta-regression analysis was performed on the study duration, sample size, cancer staging, prior breast cancer treatment, different types of TCC intervention group, different types of control group, intervention duration, mean age, and ethnicity to detect important factors leading to heterogeneity. Results: Significant heterogeneity was present in the included studies that used TCC to improve the overall quality of life of postoperative breast cancer patients from the retrieved literature $(\mathrm{P}<0.05)$. The univariate meta-regression analysis indicated that the source of heterogeneity was not apparent in the analyzed factors within or between studies. Conclusions: A normative approach in studies evaluating the use of TCC for improving the overall quality of life of postoperative breast cancer patients was not sufficiently explored in the existing randomized controlled trials (RCTs). An insufficient number of RCTs in this field may be the reason that the effect size had unclear clinical significance. The existing evidence provided limited judgment on sources of heterogeneity between studies evaluating the use of TCC for improving overall quality of life in postoperative breast cancer patients.
\end{abstract}

Keywords: Breast Neoplasms, Heterogeneity, Quality of Life, Tai Chi Chuan 


\section{Introduction}

Breast cancer is one of the most common malignant tumors worldwide in women. Between the years 2010 and 2012, the lifetime probability of developing female breast cancer was $12.3 \%$, or approximately 1 in 8 . Worldwide, breast cancer is the most common cancer in women [1].

Breast cancer treatment has developed from a solely surgical therapy to its current form as a multidisciplinary treatment. Early diagnosis and comprehensive treatment, including complementary and alternative medicine, can significantly reduce breast cancer mortality and significantly improve the prognosis of breast cancer patients [2]. While recent clinical guidelines strongly recommend some form of complementary alternative medicine (CAM) as a therapeutic option, recommendations by The National Institute for Health and Clinical Excellence (NICE) tend to exercise caution and usually stress the lack of evidence on CAM [3].

Of the 65 clinical guidelines, 48 did not mention CAM in any form, such as Tai Chi Chuan (TCC). CAM was used extensively to treat breast cancer in past decades, but evidence-based recommendations are either lacking or provide no clear evidence of the efficacy of TCC in breast cancer [3]. The guidelines for delivering therapeutic TCC for health concerns must consider multiple factors, such as the type, dose, frequency, total TCC duration, and the method used to facilitate its practice. When applying this novel, integrative aerobic practice clinically, major problems include insufficient evidence of its effect, a lack of clinical use because of physician bias, the internal coherence of each TCC style, the diverse population of breast cancer patients, and the practical concerns of senior instructors. Heterogeneity originating from clinical, methodological, and statistical sources may be an artificial consequence of an inappropriate choice of effect measure, and it is difficult to summarize the evidence on the therapeutic efficacy of TCC.

Heterogeneity of TCC is universal among the previous studies. In previous meta-analyses, data obtained from random effects models were consolidated despite large heterogeneity, the reliability of analysis results may be overestimated or underestimate. To understand the sources of the heterogeneity further, a meta-regression analysis was used to detect factors affecting heterogeneity based on factors within and between studies, which illustrated the characteristics guiding the use of TCC as a complementary and alternative treatment for patients with breast cancer.

\section{Methods}

\subsection{Search Strategy and Study Selection Criteria}

The following databases were searched from their inception through May 2017: PubMed, EMBASE, Cochrane Library, and Web of Science databases using the following search string: ("breast neoplasms" [MeSH Terms] OR "breast neoplasm" [Title/Abstract] OR "breast cancer" [Title/Abstract] OR "breast tumor" [Title/Abstract] OR "breast neoplasms" [Title/Abstract] OR "breast cancers" [Title/Abstract] OR "breast tumors" [Title/Abstract]) AND ("TCC" [MeSH Terms] OR "TCC" [Title/Abstract]) AND ("Tai-ji”" [MeSH Terms] OR "Tai-ji” [Title/Abstract]) AND ("Tai Chi”" [MeSH Terms] OR "Tai Chi” [Title/Abstract]) AND ("Tai Ji Quan" [MeSH Terms] OR "Tai Ji Quan" [Title/Abstract]) AND ("Taiji" [MeSH Terms] OR "Taiji” [Title/Abstract]) AND ("Taijiquan" [MeSH Terms] OR "Taijiquan" [Title/Abstract]) AND (random* OR "Clinical Trials as Topic" [Mesh] OR "Clinical Trial" [Publication Type]). Only English-language trials were included. Women who had been diagnosed with breast cancer, aged 18 years or older, and received active breast cancer treatments were included.

Randomized controlled trials (RCTs) were included if they examined the effects of TCC practice on general health-related quality of life, the physician permitted fitness testing and exercise, and there were no physical limitations prohibiting exercise.

\subsection{Quality Assessment and Data Extraction}

The methodological qualities of the studies were assessed independently by four reviewers according to the criteria stated in The Cochrane Collaboration Handbook (Version 2011) [4]. Disagreements were resolved by discussion with a fourth reviewer. Studies were excluded if they did not provide the post-study data required to calculate an effect size (standardized mean differences [SMDs]). If there were multiple assessment time points, the post-intervention time point was chosen. When no standard deviations were available, they were calculated from standard errors, confidence intervals, or $t$ values, or attempts were made to obtain the missing data from the authors of the trial studies by e-mail. When changes in scores and standard deviations (SDs) were not obtainable, the study was excluded. During the data analysis, effect sizes were extracted and heterogeneity factors were recorded. Factors within studies included sample size, cancer staging, previous breast cancer treatment, inclusion criteria for the Tai Chi intervention group, intervention mentors, intervention time, specific Tai Chi intervention program, establishment of a control group, and assessment tools for quality of life. Factors between studies included mean age and ethnicity.

\subsection{Statistical Analysis}

Meta-analyses were carried out using Stata software (Version 10.0, Stata Corp., College Station, TX, USA) [5]. If there was no statistically significant heterogeneity in a given data set, then a fixed effects model was used for the meta-analysis. A meta-regression approach was adopted to explore possible sources of heterogeneity among the RCTs, and heterogeneities were estimated using Cochran's Q-test, with $\mathrm{P}<0.05$ indicating a statistically significant heterogeneity.

A separate univariate meta-regression analysis was conducted on nine factors. Within studies, the factors were study time, sample size, cancer staging, prior breast cancer treatment, the Tai Chi intervention group, control group, and 
intervention duration. Factors evaluated for an impact on heterogeneity between studies included mean age and ethnicity. Because a univariate meta-regression analysis was used to detect factors underlying heterogeneity, the significance level $\alpha$ was increased to 0.1 to avoid missing important factors. A meta-regression analysis was conducted to detect significant heterogeneity factors, which were then used in the subgroup analysis. Publication bias was tested by Begg's test and Egger's test, and $\mathrm{P}>0.1$ indicated no publication bias in the retrieved literature.

\section{Results}

\subsection{Study Descriptions}

A total of 93 studies were identified using the abovementioned search strategy, 28 duplicate studies and 57 studies that did not meet the inclusion criteria were subsequently excluded. The baseline parameters of each trial were comparable; thus, six RCTs were included in the meta-analysis [6-11) (Figure 1).

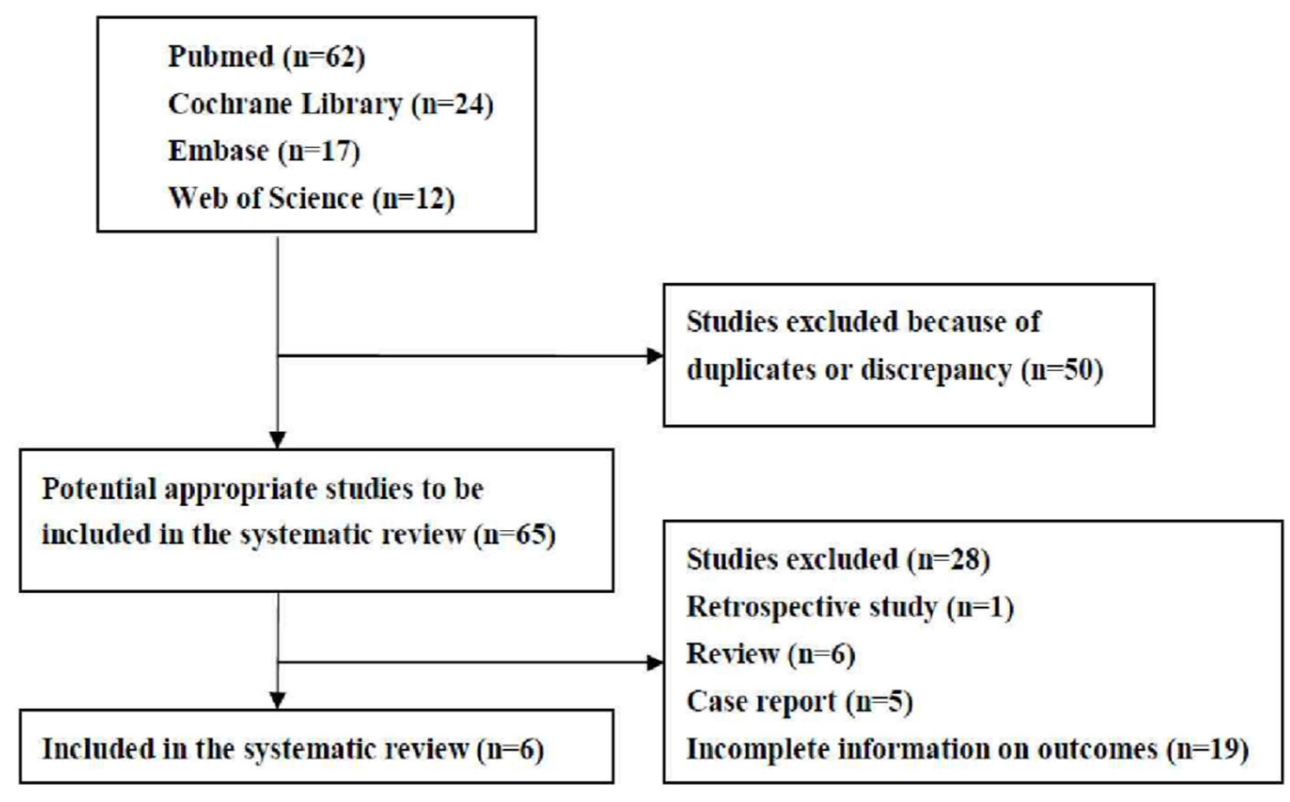

Figure 1. Flowchart of the results of the literature search.

\subsection{Clinical Heterogeneity}

All six RCTs originated from the United States of America [6-11]. The participants' ages in all studies ranged from 49 to 65 years. One RCT [11] included patients with stage I-II breast cancer, one RCT [6] included patients with stage 0-III, one RCT [7] included patients with stage I-IIIa, and two RCTs $[8,9]$ included patients with stage I-IIIb disease. Participants in two RCTs [6, 9] underwent surgery, adjuvant radiation, and/or chemotherapy during the TCC intervention. Participants in two RCTs [8, 10] underwent surgery, adjuvant radiation, chemotherapy, and hormone replacement therapy; participants in one RCT [7] underwent chemotherapy before and after enrollment; and in one RCT [1], participants underwent surgery and chemotherapy (Table 1).

In two studies, $75 \%$ of the enrolled patients were Caucasian, and $25 \%$ were identified as African-American; in one study, $90.3 \%$ of the enrolled patients were Caucasian, and $9.7 \%$ were African-American [6]. In one study, $97.3 \%$ of the enrolled patients in the Tai Chi group were Caucasian, and 3\% were other ethnicities; in the control group, $100 \%$ of the enrolled patients were Caucasian [10]. In two studies, Caucasian patients comprised $100 \%$ and $90 \%$ of the enrolled patients, respectively.

Two RCTs reported a mean body mass index (BMI) of 26.3 $\pm 4.9 \mathrm{~kg} / \mathrm{m}^{2}[6,8]$. In one RCT, the mean BMI of patients in the Tai Chi group was $24.89 \pm 1.93 \mathrm{~kg} / \mathrm{m}^{2}$, while that of the control group was $24.97 \pm 1.39 \mathrm{~kg} / \mathrm{m}^{2}$. The mean BMI was not stated in the other studies (Table 2).

Two RCTs $[6,8]$ based their standard Tai Chi regimen on the American College of Sports Medicine guidelines; published literature was used as the basis in two RCTs [7, 9], and the other studies did not indicate the inclusion criteria. The intensity of the TCC regimens varied, but overall, sessions were performed three times weekly, lasted between 60 and 90 minutes each, and the treatment duration lasted from 3 weeks to 6 months. The TCC interventions were heterogeneous and included regimens using basic Chi Kung [6, $8]$, integrated TCC regimens [10, 11], five-element regimens [7], and Yang-style TCC [9]

In two RCTs, participants in the control group underwent a psychosocial therapy intervention $[6,8]$, and in the remaining RCTs, the control group underwent health education [10], spiritual growth counseling [11], Spiegel's supportive-expressive therapy [9], or standard care [7) (Table 2). 
Table 1. Characteristics of the included studies.

\begin{tabular}{|c|c|c|c|c|c|}
\hline \multirow{2}{*}{ Authors/year/country } & \multicolumn{2}{|l|}{ No. of patients } & \multicolumn{2}{|l|}{ Mean age (y) } & \multirow{2}{*}{ Status of cancer } \\
\hline & Tai Chi Chuan & Control group & Tai Chi Chuan & Control group & \\
\hline \multirow[t]{2}{*}{ Campo RA et al., 2013 [10] USA } & 29 & 25 & $65.64 \pm 7.15$ & $66.54 \pm 7.15$ & I-III \\
\hline & & & & & $\begin{array}{l}0-\text { III: } \\
0(7.0 \%)\end{array}$ \\
\hline \multirow[t]{3}{*}{ Mustian KM et al., 2004 [6] USA } & 11 & 10 & $52 \pm 9$ & $52 \pm 9$ & I $(52.7 \%)$ \\
\hline & & & & & II $(34 \%)$ \\
\hline & & & & & III $(7 \%)$ \\
\hline Mustian KM et al., 2008 [8] USA & 11 & 10 & $52 \pm 9$ & Unclear & I-IIIb \\
\hline Rausch SM et al., 2007 [11] USA & 15 & 14 & $49(33-69)$ & $49(33-69)$ & I-II \\
\hline Robins JL et al., 2013 [7] USA & 37 & 36 & 50 & 50 & I-IIIa \\
\hline Sprod LK et al., 2012 [9] USA & 9 & 10 & $54.33 \pm 3.55$ & $52.7 \pm 2.11$ & $\mathrm{I}-\mathrm{IIIb}$ \\
\hline
\end{tabular}

Table 1. Continued.

\begin{tabular}{|c|c|c|c|c|}
\hline \multirow{2}{*}{ Authors/year/country } & \multirow{2}{*}{ Current treatment } & \multicolumn{2}{|l|}{ Race } & \multirow{2}{*}{ BMI $\left(\mathbf{k g} / \mathbf{m}^{2}\right)$} \\
\hline & & Tai Chi Chuan & Control group & \\
\hline $\begin{array}{l}\text { Campo RA et al., } 2013 \\
\text { [10] USA }\end{array}$ & $\begin{array}{l}\text { Treatment completed over } 3 \text { months before enrollment; patients } \\
\text { underwent surgery, radiation, chemotherapy, and hormonal } \\
\text { therapy }\end{array}$ & $\begin{array}{l}\text { Caucasian, } 97.3 \% \\
\text { Non-white, } 3 \%\end{array}$ & Caucasian, $100 \%$ & Not mentioned \\
\hline $\begin{array}{l}\text { Mustian KM et al., } \\
2004 \text { [6] USA }\end{array}$ & $\begin{array}{l}\text { Treatment completed } 36 \text { months before enrollment; patients } \\
\text { underwent surgical treatment }(100 \%) \text {, adjuvant radiation } \\
(61 \%) \text {, and/or chemotherapy }(84 \%)\end{array}$ & \multicolumn{2}{|c|}{$\begin{array}{l}\text { Caucasian, } 90.3 \% \text {; African-American or } \\
\text { black, } 9.7 \%\end{array}$} & $26.3 \pm 4.9$ \\
\hline $\begin{array}{l}\text { Mustian KM et al., } \\
2008 \text { [8] USA }\end{array}$ & $\begin{array}{l}\text { Treatment completed more than } 1 \text { month but less than } 30 \\
\text { months before enrollment; patients underwent surgery }(100 \%) \text {, } \\
\text { adjuvant radiation }(61 \%) \text {, and hormonal therapy }(56 \%)\end{array}$ & \multicolumn{2}{|l|}{ Caucasian, $90 \%$} & $26.3 \pm 4.9$ \\
\hline $\begin{array}{l}\text { Rausch SM et al., } 2007 \\
\text { [11] USA }\end{array}$ & $\begin{array}{l}\text { Treatment completed more than } 1 \text { month previously, but less } \\
\text { than } 30 \text { months before enrollment; patients underwent surgery } \\
\text { and chemotherapy }\end{array}$ & \multicolumn{2}{|c|}{$\begin{array}{l}\text { Caucasian, } 73 \% \text {; African-American or } \\
\text { black, } 27 \%\end{array}$} & Not mentioned \\
\hline $\begin{array}{l}\text { Robins JL et al., } 2013 \\
\text { [7] USA }\end{array}$ & Underwent chemotherapy before and after enrollment & \multicolumn{2}{|c|}{$\begin{array}{l}\text { Caucasian, } 75 \% \text {; African-American or } \\
\text { black, } 25 \%\end{array}$} & Not mentioned \\
\hline $\begin{array}{l}\text { Sprod LK et al., } 201 \\
\text { [9] USA }\end{array}$ & $\begin{array}{l}\text { Treatment completed more than } 1 \text { month but less than } 30 \\
\text { months before enrollment; underwent surgery ( } 100 \%) \text {, adjuvant } \\
\text { radiation }(47 \%) \text {, and/or chemotherapy }(89 \%)\end{array}$ & Caucasian, $100 \%$ & Caucasian, $100 \%$ & $\begin{array}{l}24.89 \pm 1.93 \\
(\mathrm{TCC}) 24.97 \pm \\
1.39 \text { (control) }\end{array}$ \\
\hline
\end{tabular}

Table 2. Methodological quality of the included studies.

\begin{tabular}{|c|c|c|c|}
\hline Reference & Tai Chi Chuan inclusion criteria & Tai Chi Chuan instructor & Duration \\
\hline Campo RA et al., 2013 [10] USA & Not mentioned & $\begin{array}{l}\text { Experienced } \\
\text { instructor }\end{array}$ & $\begin{array}{l}60 \mathrm{~min} \text {, three times weekly for } 12 \\
\text { weeks }\end{array}$ \\
\hline Mustian KM et al., 2004 [6] USA & $\begin{array}{l}\text { American College of Sports } \\
\text { Medicine guidelines }\end{array}$ & $\begin{array}{l}\text { American College of Sports Medicine } \\
\text { certified health and fitness instructor }\end{array}$ & $\begin{array}{l}60 \text { min, three times weekly for } 3 \\
\text { months }\end{array}$ \\
\hline Mustian KM et al., 2008 [8] USA & $\begin{array}{l}\text { American College of Sports } \\
\text { Medicine guidelines }\end{array}$ & $\begin{array}{l}\text { American College of Sports Medicine } \\
\text { certified health and fitness instructor }\end{array}$ & $\begin{array}{l}60 \mathrm{~min} \text {, three times weekly } \\
\text { for } 12 \text { weeks }\end{array}$ \\
\hline Rausch SM et al., 2007 [11] USA & Not mentioned & $\begin{array}{l}\text { Experienced member of the sponsor's } \\
\text { current research team }\end{array}$ & $\begin{array}{l}60 \mathrm{~min} \text { at first, } 90 \mathrm{~min} \text { from the second } \\
\text { session, and once weekly for } 10 \text { weeks }\end{array}$ \\
\hline Robins JL et al., 2013 [7] USA & $\begin{array}{l}\text { Referring to the published } \\
\text { literature }\end{array}$ & TCC training videotapes/DVDs & $\begin{array}{l}90 \text { min } \\
\text { each week for a total of } 6 \text { months }\end{array}$ \\
\hline Sprod LK et al., 2012 [9] USA & $\begin{array}{l}\text { Referring to the published } \\
\text { literature }\end{array}$ & $\begin{array}{l}\text { American College of Sports Medicine } \\
\text { certified health and fitness instructor }\end{array}$ & $\begin{array}{l}60 \mathrm{~min} \text {, three times per week for } 12 \\
\text { weeks }\end{array}$ \\
\hline
\end{tabular}

Table 2. Continued.

\begin{tabular}{|c|c|c|c|}
\hline Reference & Tai Chi Chuan regimen & Control group & Outcome measures/results \\
\hline $\begin{array}{l}\text { Campo RA et al., } \\
2013 \text { [10] USA }\end{array}$ & $\begin{array}{l}\text { 20-min warm-up that consisted of seated } \\
\text { meditation, self-massage of acupressure points, } \\
\text { and light stretching, followed by } 30 \text { min of TCC } \\
\text { movements and } 10 \text { min of closing movements. } \\
\text { TCC consisted of } 19 \text { simple, repetitive, } \\
\text { non-strenuous movements and } 1 \text { standing pose }\end{array}$ & $\begin{array}{l}\text { Health education control: } 60 \text {-min health } \\
\text { education sessions focused on topics } \\
\text { relevant to aging (e.g., successful aging, } \\
\text { pain, nutrition, sleep changes, social } \\
\text { roles, and relationships); three times a } \\
\text { week over } 12 \text { weeks }\end{array}$ & $\begin{array}{l}\text { Significant improvements in mental } \\
\text { and physical benefits (SF-36 Health } \\
\text { Survey) }(\mathrm{P}=0.01)\end{array}$ \\
\hline $\begin{array}{l}\text { Mustian KM et al., } \\
2004 \text { [6] USA }\end{array}$ & $\begin{array}{l}\text { Participants performed } 10 \text { min of warm-up } \\
\text { stretching and basic Chi Kung (stationary TCC } \\
\text { fundamentals). The participants then performed } \\
\text { TCC for approximately } 40 \text { min, and learned a } \\
\text { 15-move short form of Yang-style TCC. During the }\end{array}$ & $\begin{array}{l}\text { Psychosocial therapy control: an } \\
\text { open-ended format that placed a strong } \\
\text { emphasis on teaching behavioral coping } \\
\text { strategies, peer support, and group } \\
\text { cohesion ( } 60 \text { min, three times weekly }\end{array}$ & $\begin{array}{l}\text { Significant improvements in both } \\
\text { quality of life (FACIT-F) }(\mathrm{P}=0.00) \\
\text { and self-esteem }(\text { Rosenberg } \\
\text { Self-Esteem Scale) }(\mathrm{P}<0.01)\end{array}$ \\
\hline
\end{tabular}




\begin{tabular}{|c|c|c|c|}
\hline Reference & Tai Chi Chuan regimen & Control group & Outcome measures/results \\
\hline $\begin{array}{l}\text { Mustian KM et al., } \\
2008 \text { [8] USA }\end{array}$ & $\begin{array}{l}\text { last } 10 \text { min of each session, participants were } \\
\text { instructed in regulatory breathing, imagery, and } \\
\text { meditation to enhance their TCC skills and provide } \\
\text { an exercise cool-down. } \\
10 \text { min of warm-up stretching and basic Chi Kung } \\
\text { (stationary TCC fundamentals); TCC for } 40 \text { min, } \\
\text { and a } 15 \text {-move short-form sequence of Yang-style } \\
\text { TCC and a 104-move Yang-style long form. } \\
\text { During the final } 10 \text { min of each session, } \\
\text { participants were instructed in regulatory } \\
\text { breathing, imagery, and meditation to enhance their } \\
\text { TCC skills and provide an exercise cool-down. }\end{array}$ & $\begin{array}{l}\text { Psychosocial therapy control: an } \\
\text { open-ended format that placed a strong } \\
\text { emphasis on teaching behavioral coping } \\
\text { strategies, peer support, and group } \\
\text { cohesion ( } 60 \text { min, three times weekly } \\
\text { for } 12 \text { weeks }\end{array}$ & $\begin{array}{l}\text { Significant improvement in quality } \\
\text { of life }(\mathrm{FACIT})(\mathrm{P}=0.03) \text {, but not in } \\
\text { the control group }(\mathrm{P}>0.05)\end{array}$ \\
\hline $\begin{array}{l}\text { Rausch SM et al., } \\
2007 \text { [11] USA }\end{array}$ & Short form of TCC involving eight movements & $\begin{array}{l}\text { Spiritual growth control: designed for } \\
\text { personal exploration and communal } \\
\text { sharing by participating in activities } \\
\text { designed to facilitate an understanding } \\
\text { and appreciation of spirituality }\end{array}$ & $\begin{array}{l}\text { Significant improvement in } \\
\text { perceived stress (Impact of Events } \\
\text { Scale) }(\mathrm{P}<0.05) \text {; significant } \\
\text { improvement in quality of life } \\
\text { (FACT-B) }(\mathrm{P}<0.01)\end{array}$ \\
\hline $\begin{array}{l}\text { Robins JL et al., } \\
2013 \text { [7] USA }\end{array}$ & $\begin{array}{l}\text { Eight TCC movements focused on repetition, } \\
\text { attention, flexibility, and mind-body relaxation and } \\
\text { a cognitive "five elements" component to enhance } \\
\text { stress management. }\end{array}$ & $\begin{array}{l}\text { Usual care control: personal exploration } \\
\text { and group sharing of spirituality, aimed } \\
\text { at enhancing awareness of the meaning } \\
\text { and expression of spirituality while } \\
\text { supporting both secular and religious } \\
\text { views of spirituality in a group format. }\end{array}$ & $\begin{array}{l}\text { Significant improvement in } \\
\text { perceived stress scores (Impact of } \\
\text { Events Scale) }(\mathrm{P}<0.0001) \text { and } \\
\text { quality of life (FACT-B) }(\mathrm{P}< \\
0.0001) \text { in the intervention group }\end{array}$ \\
\hline $\begin{array}{l}\text { Sprod LK et al., } \\
2012 \text { [9] USA }\end{array}$ & $\begin{array}{l}10 \text {-min warm-up, } 40 \text { min of Yang-style TCC using } \\
\text { a } 15 \text {-move short form, which were the traditional } \\
15 \text { moves, a } 104 \text {-move long form, and } 10 \text { min of } \\
\text { breathing, imagery, and meditation. }\end{array}$ & $\begin{array}{l}\text { Psychosocial therapy: Spiegel's } \\
\text { supportive-expressive group therapy } \\
\text { model was followed, and an emphasis } \\
\text { was placed on behavioral coping skills, } \\
\text { group cohesion, and peer support. }\end{array}$ & $\begin{array}{l}\text { Significant improvement in quality } \\
\text { of life, physical functioning } \\
\text { (HRQOL) }(\mathrm{P}=0.030) \text {, physical role } \\
\text { limitations (HRQOL) }(\mathrm{P}=0.023) \text {, } \\
\text { and social functioning (HRQOL) (P } \\
=0.020 \text { ) }\end{array}$ \\
\hline
\end{tabular}

Table 3. Continued.

\begin{tabular}{|c|c|c|c|c|c|c|}
\hline Reference & Randomization & $\begin{array}{l}\text { Allocation } \\
\text { concealment }\end{array}$ & Blinding & $\begin{array}{l}\text { Incomplete } \\
\text { outcome } \\
\text { data }\end{array}$ & $\begin{array}{l}\text { Selective } \\
\text { outcome } \\
\text { reporting }\end{array}$ & $\begin{array}{l}\text { Other } \\
\text { sources } \\
\text { of bias }\end{array}$ \\
\hline Campo RA et al., 2013 [10] USA & $\begin{array}{l}\text { Randomized using random } \\
\text { permuted blocks }\end{array}$ & Mentioned & $\begin{array}{l}\text { Yes } \\
\text { (statisticians) }\end{array}$ & Yes & Unclear & Unclear \\
\hline Mustian KM et al., 2004 [6] USA & Flipping a coin & Mentioned & Mentioned & Unclear & Unclear & Unclear \\
\hline Mustian KM et al., 2008 [8] USA & Flipping a coin & Mentioned & Mentioned & Unclear & Unclear & Unclear \\
\hline Rausch SM et al., 2007 [11] USA & Randomized via mailed letters & $\begin{array}{l}\text { Opaque, numbered } \\
\text { envelopes }\end{array}$ & Mentioned & Unclear & Unclear & Unclear \\
\hline Robins JL et al., 2013 [7] USA & $\begin{array}{l}\text { Randomized using a } \\
\text { computer-generated random list }\end{array}$ & Mentioned & Yes (assessor) & Yes & Unclear & Unclear \\
\hline Sprod LK et al., 2012 [9] USA & Flipping a coin & Mentioned & Mentioned & No & Unclear & Unclear \\
\hline
\end{tabular}

Table 4. Effect sizes of the meta-regression analysis for overall quality of life.

\begin{tabular}{|c|c|c|c|c|c|c|c|}
\hline & \multirow{2}{*}{ Constant } & \multirow{2}{*}{ Coefficient } & \multirow{2}{*}{ SE } & \multirow{2}{*}{ T-value } & \multirow{2}{*}{ P-value } & \multicolumn{2}{|c|}{$\mathbf{9 5 \%}$ confidence interval } \\
\hline & & & & & & $\mathbf{U L}$ & $\mathbf{L L}$ \\
\hline \multicolumn{8}{|c|}{ General health-related quality of life } \\
\hline Age & -0.45 & 0.20 & 0.32 & 0.61 & 0.57 & -0.71 & 1.11 \\
\hline Stage of cancer & -0.34 & 0.82 & 0.26 & 0.31 & 0.76 & -0.65 & 0.81 \\
\hline Current treatment & -0.06 & -0.05 & 0.15 & -0.35 & 0.75 & -0.48 & 0.38 \\
\hline Race & 0.07 & -0.06 & 0.20 & -0.34 & 0.75 & -0.620 & 0.48 \\
\hline BMI & 0.75 & -0.36 & 0.20 & -1.78 & 0.19 & -0.94 & 0.20 \\
\hline Tai Chi Chuan inclusion criteria & 0.16 & -0.11 & 0.20 & 0.55 & 0.60 & -0.67 & 0.45 \\
\hline Tai Chi Chuan instructor & -0.14 & 0.00 & 0.28 & 0.02 & 0.99 & -0.78 & 0.79 \\
\hline Duration & -0.12 & -0.00 & 0.36 & -0.02 & 0.98 & -1.02 & 1.00 \\
\hline Tai Chi Chuan movements & 0.16 & -0.11 & 0.20 & -0.55 & 0.61 & -0.67 & 0.45 \\
\hline Control group & 0.16 & -0.11 & 0.20 & -0.55 & 0.61 & -0.67 & 0.45 \\
\hline Scale & -0.23 & 0.03 & 0.20 & 0.18 & 0.87 & -0.51 & 0.59 \\
\hline Bias & & -2.46 & 2.49 & -0.99 & 0.37 & -9.34 & 4.43 \\
\hline
\end{tabular}




\subsection{Methodological Heterogeneity}

All six RCTs [6-11] described a method of adequate random sequence generation and allocation concealment. The blocks in one RCT [11] were concealed, and sequences were stored in sealed, opaque, and numbered envelopes; one RCT [10] adopted blinding of the statisticians to the patient allocations, and one RCT [7] reported blinding of the assessors to the patient allocations (Table 3).

The remaining RCTs did not indicate whether selective outcome reporting or other sources of bias were present. Generally, when assessing the risk of bias by the Cochrane Book Scale, the methodological quality of the trials was higher in measures of similarity between the groups at baseline, in studies with less than a $15 \%$ dropout rate, and in between-group statistical comparisons, measures, and variability data (Table 3).

\subsection{Effect Size of Tai Chi Chuan on General Health-Related Quality of Life}

The Functional Assessment of Cancer Therapy-Breast (FACT-B) [7, 11], Functional Assessment of Chronic Illness Therapy-Fatigue survey (FACIT) [8], Health-Related Quality of Life 36 (HRQOL) [9], and Health-Related Quality of Life 36 (SF-36) [10] were used to assess the outcomes of general health-related quality of life in the six RCTs. Comparisons of the studies revealed substantial heterogeneity $\left(\mathrm{P}=0.05, \mathrm{I}^{2}=\right.$ $53.7 \%)$. The pooled results suggested that general health-related quality of life failed to improve in the TCC group compared to the control group $(\mathrm{SMD}=0.24,95 \% \mathrm{CI}=$ $-0.03,0.51 ; \mathrm{P}=0.08)$ (Figure 2).

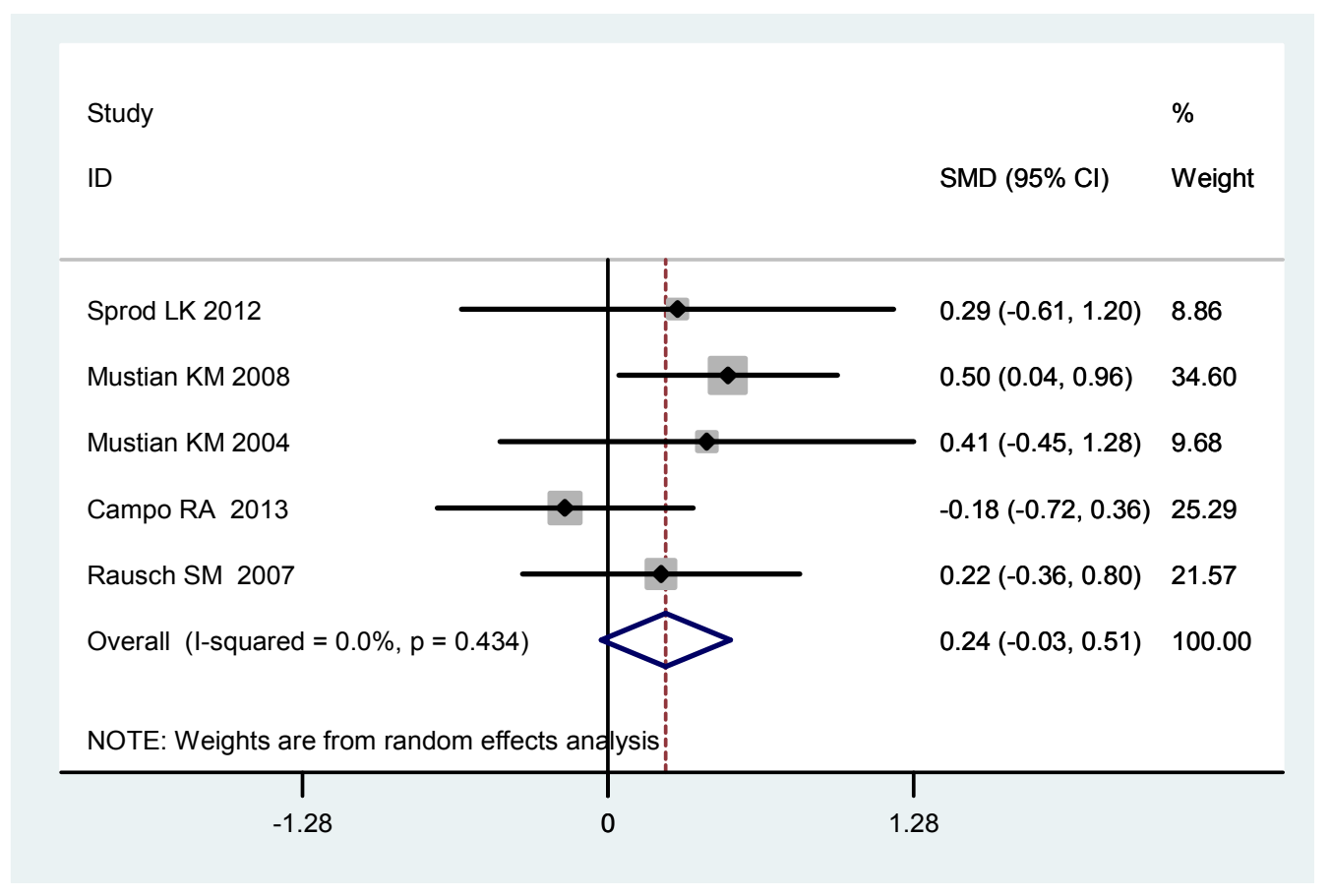

Figure 2. Physical well-being.

\subsection{Meta-regression and Publication Bias Test}

A separate univariate meta-regression analysis was conducted on 11 factors within and between studies (sample size, cancer staging, prior treatments, ethnicity, BMI, inclusion criteria for the Tai Chi intervention group, intervention mentors, intervention duration, Tai Chi intervention program, establishment of a control group, and quality of life assessment tools) to evaluate their impacts on heterogeneity between studies (Table 4). The results showed that the $\mathrm{P}$ values for all 11 factors were greater than 0.5 , and no source of heterogeneity was found for factors within or between studies. Publication bias was tested by Begg's test and Egger's test, and the resulting P values were greater than 0.05 . The funnel plot was generally symmetrical, and there were fewer chances of publication bias in the retrieved literature (Figure 3-5).

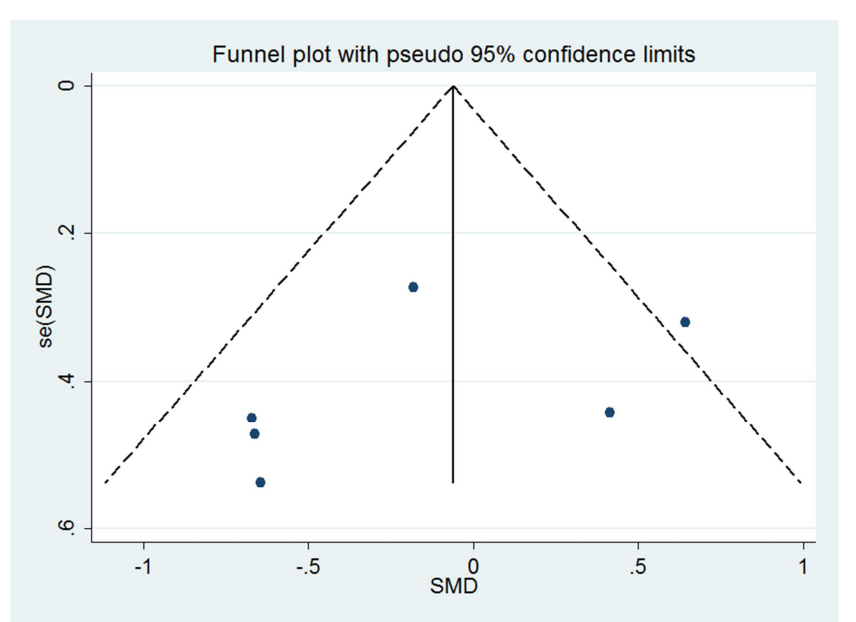

Figure 3. A funnel plot of the overall quality of life of patients with breast cancer with a Tai Chi Chuan intervention. 
The change in overall quality of life due to the Tai Chi Chuan intervention (x-axis) is plotted against the SE of the change in overall quality of life (y-axis) for each trial. The vertical line represents the overall pooled fixed-effect estimate, and the dashed lines indicate the expected $95 \%$ CI for a given SE.

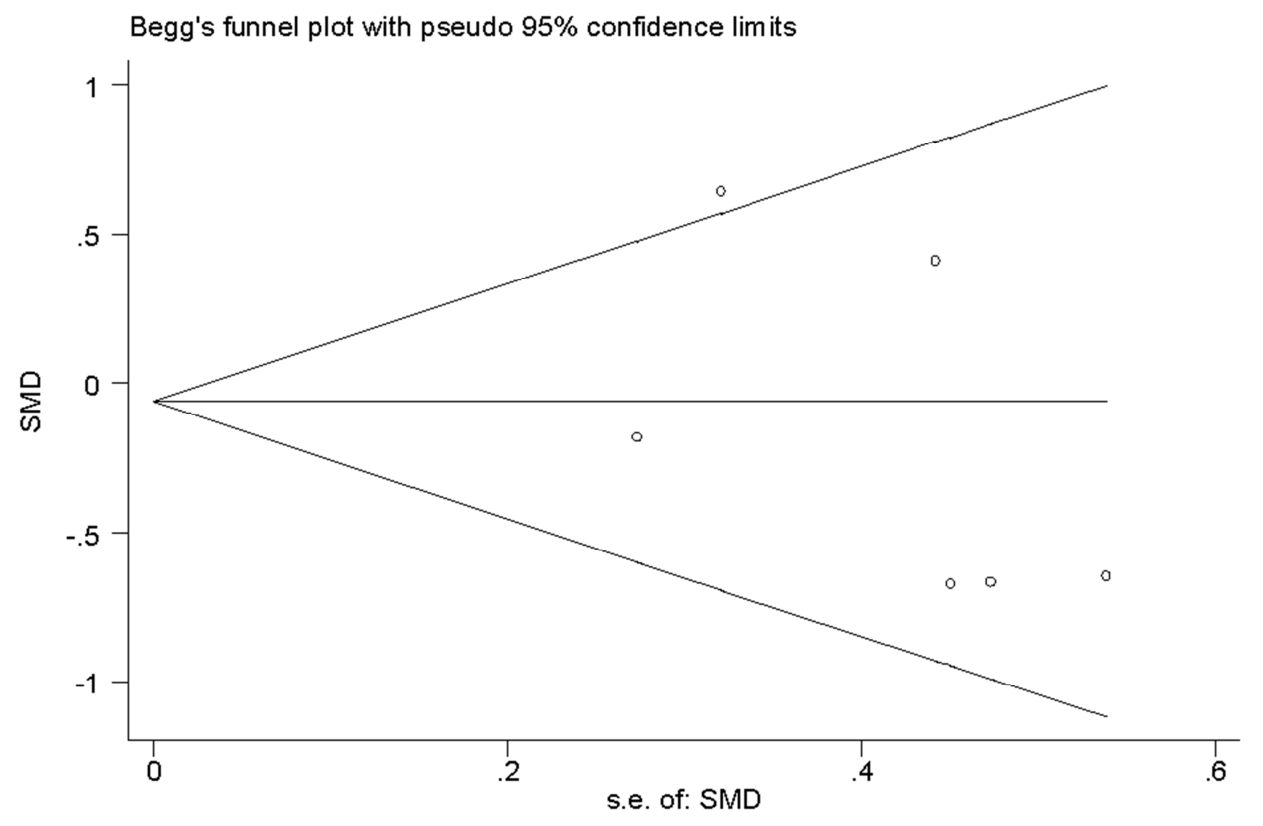

Figure 4. A Begg's funnel plot for the meta-analysis of the overall quality of life of patients with breast cancer with a Tai Chi Chuan intervention.

Each point represents a separate study for the indicated correlation. Vertical line: SMD, standardized mean difference, natural logarithm of the relative risk (RR); horizontal line: SE, standard error, mean magnitude of the effect.

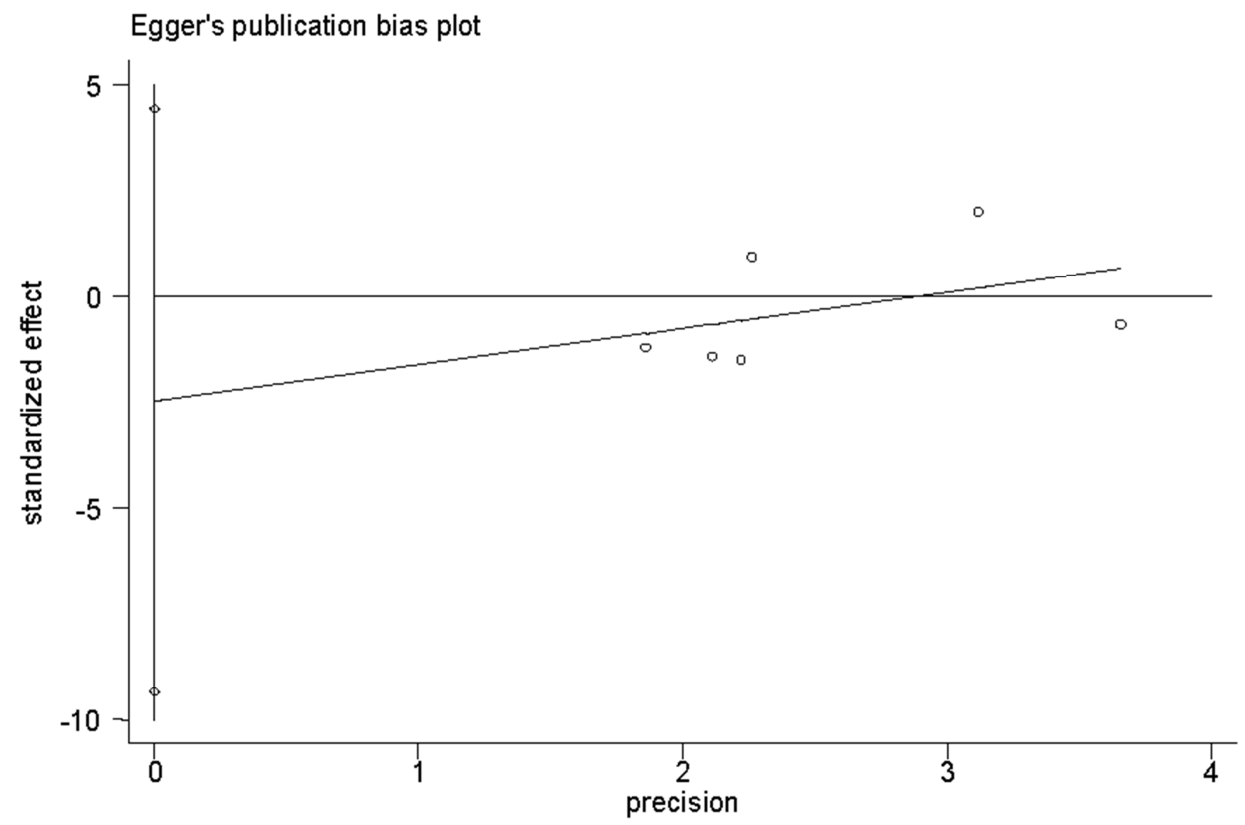

Figure 5. An Egger's funnel plot for the meta-analysis of the overall quality of life of patients with breast cancer with a Tai Chi Chuan intervention.

Each point represents a separate study for the indicated correlation. Standardized effect, standard error of the natural logarithm of relative risk; horizontal line, relative risk. Vertical line: standardized effect, samples were divided by their standard error; horizontal line: precision, standard error of the reciprocal.

\section{Discussion}

The existence of heterogeneity guide the decision to conduct a combined analysis and model selection. Therefore, it is essential to conduct a systematic review or meta-analysis and a heterogeneity evaluation of RCTs [12, 13]. 
Heterogeneity may be due to genuine differences in the participants, interventions, co-interventions, outcomes, measurements, settings, and numerous other factors varying between the data sets, studies, and participants [14]. There are two sources of study heterogeneity. one is heterogeneity within studies. That is, different studies of the same population show different effects because of sampling error. The other source of heterogeneity is variant between studies, which refers to different effects resulting from distinctions among the study sample and population, or differences in bias control [15].

This study performed a univariate meta-regression analysis to identify factors within and between studies, the results did not show any significant factor leading to heterogeneity. The evidence, which was collected from previously published systematic reviews and RCTs on Tai Chi to manage overall quality of life in postoperative breast cancer patients, suggested that Tai Chi did not significantly affect quality of life compared to conventional rehabilitation, psychological support therapy, or standard treatment [16-18]. However, three RCTs showed that Tai Chi might be effective on improving the range of motion in the shoulder, aerobic capacity, and especially shoulder flexion, extension, abduction, rotation, and muscle strength in postoperative patients with breast cancer. All results showed better outcomes in the Tai Chi intervention group than the control. However, differences in the enrolled subjects, intervention programs, and evaluation methods led to higher heterogeneity between the studies, and a combined effect analysis could not be conducted. The quality and quantity of research were insufficient and could not strongly demonstrate the actual clinical effectiveness of the Tai Chi intervention.

Meta-regression is an extension of the subgroup analysis; a multivariate analysis can be performed, and the number of included studies should be at least 10 generally [15]. Unfortunately, there were fewer than five study samples in previously published TCC RCTs and systematic reviews in patients with breast cancer. Because small sample studies were included, the effects of the meta-regression and funnel plot analysis used to assess publication bias were relatively limited, the risk of publication bias of systematic review based on the RCTs with a small sample may be greater. Whether the result is positive or negative (i.e., whether the difference between the Tai Chi intervention and control groups showed a statistically significant improvement in the quality of life of postoperative breast cancer patients), these RCTs may not provide an adequately precise estimate of efficacy.

In this study, 11 common factors causing clinical and methodological heterogeneity were analyzed. Based on small sample of included studies, the accuracy of the conclusions could be potentially affected. When only a few pilot studies are available, the completed systematic review may overestimate or underestimate the effect size. If negative studies are based on a small sample size, the results are especially questionable. The funnel plot in this study suggested that there might be no publication bias. However, the main factors leading to publication bias are related to several parameters, such as the statistical significance of the treatment effect size, sample size, innovativeness of the treatment options, importance of the studied objectives, and research quality. The number of studies required to generate a funnel plot is large, but in this study, only six RCTs were included. A symmetrical funnel plot may have appeared by chance because of an insufficient number of data points, and the result should be interpreted cautiously.

Based on the included studies, a normative approach did not receive sufficient attention in the existing RCTs, and only 2 RCTs adopted blinding of the statisticians/assessors to the patient allocations [7, 10]. Evidence of missing blinding would affect the critical appraisal and the reliability of the findings [19]. Among the included studies, only one RCT divulged its method for obscuring the random assignments [11], and the others made no statement.

The reasons for incomplete data were not reported in all of the included studies, and thus, the sample sizes in the studies were not calculated using scientific methods; as a result, bias may have occurred because of an insufficient sample size. In all of the included studies, only two RCTs showed high homogeneity, and the protocol of TCC in the other RCTs were quite different. Based on small sample size, the existing source of heterogeneity does not show convincingly that tai chi is effective or invalid in patients with breast cancer. Tai Chi is rooted in East Asian culture and has traditional characteristics, and there are numerous recipients and believers in the philosophy of psychosomatic therapy among postoperative breast cancer patients. Investigators in non-English speaking countries, such as China and other Asian countries, may instead submit their negative or positive studies to domestic non-English journals.

In addition, multicenter observational studies with a large sample size, case-control studies, or quasi-randomized controlled trials are considered low-quality evidence in evaluated the impact of a Tai Chi on the quality of life of postoperative breast cancer patients. These studies may be missed by a systematic review and retrieves only in English journals, and thus, the systematic review will also be subjects.

The principal aim of Tai Chi is to regulate the dynamic and static equilibrium of the human body, eliminate body tension, build a fully automated tension-relaxation mechanism, and establish a new excitation-suppression transformation process. To preserve health, Tai Chi uses movement to maintain shape and a static state of repose, thus unifying the mind and body. TCC as an intervention may provide benefit to cancer survivors in these multiple areas of need based on its characteristics of combining aspects of meditation and aerobic exercise. Various protocols of the Tai Chi exercise have been created, multiple factors such as the expertise of Tai Chi practitioners, inconsistent designs of the Tai Chi programs (frequency, intensity, content, and duration), multivariant control group and biological and sociological characteristics of breast cancer greatly affect the clinical significance of the mean effect size [20]. Consequently, these clinical and methodological factors may affect the reliability of the conclusions. We have no solid empirical evidence on the 
extent of concordance between methodological and clinical heterogeneity. TCC is widely accepted as beneficial for breast cancer patients both during and after treatment, and also used as an adjunct to mainstream breast cancer care. Unfortunately, TCC lacks a gold standard protocol for clinical treatment effects [21]. Typically, there are difficulties even establishing the main effects in medical interventions, and claims of subgroup differences are poorly examined.

Systematic reviews and meta-analyses are insufficiently informative if they are poor consistency or do not include all available current evidence. In fact, most meta-analyses have a relatively narrow scope and focus on specific treatments [22]. This descriptive study shows that the same-disease-with-different treatments phenomenon widely exists in published systematic reviews, meta-analyses, and RCTs evaluating Tai Chi for improving overall quality of life in postoperative breast cancer patients [23, 24].

Comprehensive TCC assessment is a multidimensional interdisciplinary diagnostic process used to determine the medical, psychological, and functional capabilities of patients with breast cancer. The result of heterogeneity influenced how the total estimate was obtained. In the presence of heterogeneity, random effects methods would have been used. A random effects meta-analysis would have produced a wider confidence interval for the subtotal effect than a fixed effects meta-analysis, resulting in a less accurate subtotal effect size. In addition, the insufficient number of RCTs using Tai Chi in patients with breast cancer may have been one of the essential causes underlying the insignificant clinical efficacy, meanwhile, a lack of standard protocol could result in clinical heterogeneity, the primary emphasis of this review was to provide a shortterm outcome to elucidate the value of TCC. Therefore, further trials that focus on long-term outcomes with a large sample size are needed to find potential advantages or disadvantages. In summary, RCTs evaluating the use of Tai Chi as a complementary and alternative therapy to improve overall quality of life in postoperative breast cancer patients may contain clinical and methodological heterogeneities.

\section{Conclusion}

The protocol of included randomized controlled trials of this study were diversity, methodological heterogeneity and small-study are prominent problems of Tai Chi related to systematic review and meta-analysis, in order to reduce those bias, it can be realized by expanding sample size, strengthening the standardized clinical trial design and popularizing the registration system of traditional Tai Chi.

\section{Source of Funding}

We are indebted to the following education and research institutions for financial support.

1. Key Project of the 2017 year, Vocational Technical Education Branch of Higher Education Research Academy of China: Research on the Path of the Application of Evidence-based Medical Education and
Knowledge Transformation in Higher Medical Vocational Education (Grant No: GZYZD2017012)

2. Key Project of the 2017 year, Tianjin Higher Vocational and Technical Education Research Institute: the International Standard and Path Study on the Advanced Training of JBI Health Care Model for Higher Medical Vocational Education (Grant No: XVII106)

3. Lanzhou Municipal Science and Technology Bureau of Gansu province of China of Science and technology Project (medical and health special): Applicability Evaluation of Systematic Review of Breast Cancer Treated with Non Drug Complementary Alternative Medicine (Grant No: 2016-2-68)

\section{References}

[1] Rojas K, Stuckey A. Breast Cancer Epidemiology and Risk Factors. Clin Obstet Gynecol. Vol. 59, No. 4, 2016, pp. 651-672.

[2] Subramani R, Lakshmanaswamy R. Complementary and Alternative Medicine and Breast Cancer. Prog Mol Biol Transl Sci. Vol. 151, 2017, pp. 231-274.

[3] Ernst E. Assessments of complementary and alternative medicine: the clinical guidelines from NICE. International Journal of Clinical practice. Vol. 64, No. 10, 2010, pp. 1350-1358.

[4] Higgins JPT, Green S. Cochrane Handbook for Systematic Reviews of Interventions. Version 5.1.0. Online document at: The Cochrane Collaboration http://www.07November2011.cochrane-handbook.org. Accessed 2011.

[5] Stata software (computer program) (version 10.0). Stata Corp., College Station, TX, USA. 2010.

[6] Mustian KM, Katula JA, Gill DL, Roscoe JA, Lang D, Murphy K. Tai Chi Chuan, health-related quality of life and self-esteem: a randomized trial with breast cancer survivors. Support Care Cancer. Vol. 12, No. 12, 2004, pp. 871-876.

[7] Robins JL, McCain NL, Elswick RK Jr, Walter JM, Gray DP, Tuck I. Psychoneuroimmunology-based stress management during adjuvant chemotherapy for early breast cancer. Evidence Based Complementary Alternative Medicine. 2013; 2013: 372908 .

[8] Mustian KM, Palesh OG, Flecksteiner SA. Tai Chi Chuan for breast cancer survivors. Medical Sport Science. Vol. 52, 2008, pp. 209-217.

[9] Sprod LK, Janelsins MC, Palesh OG, et al. Health-related quality of life and biomarkers in breast cancer survivors participating in Tai Chi Chuan. Journal of Cancer Survivorship. Vol. 6, 2012,pp. 146-54

[10] Campo RA, O'Connor K, Light KC, et al. Feasibility and acceptability of a Tai Chi Chih randomized controlled trial in senior female cancer survivors. Integrative Cancer Therapies. Vol. 12, No. 6, 2013, pp. 464-474.

[11] Rausch SM. Evaluating psychosocial effects of two intervention, Tai Chi and spiritual growth groups, in women with breast cancer. Dissertation, June 2007 Virginia Commonwealth University. 
[12] Choi SW, Lam DM. Heterogeneity in meta-analyses. Comparing apples and oranges? Anaesthesia. Vol. 72, No. 4, 2017, pp, 532-534.

[13] Hicks A, Fairhurst C, Torgerson DJ. A simple technique investigating baseline heterogeneity helped to eliminate potential bias in meta-analyses. J Clin Epidemiol. Vol. 95, 2018, pp. 55-62.

[14] Garcia-Alamino JM, Bankhead C, Heneghan C, Pidduck N, Perera R. Impact of heterogeneity and effect size on the estimation of the optimal information size: analysis of recently published meta-analyses. BMJ Open. Vol. 7, No. 11, 2017, pp.e015888.

[15] Doleman B, Sutton AJ, Sherwin M, Lund JN, Williams JP. Baseline Morphine Consumption May Explain Between-Study Heterogeneity in Meta-analyses of Adjuvant Analgesics and Improve Precision and Accuracy of Effect Estimates. Anesth Analg. Vol. 126 No. 2, 2018, pp. 648-660.

[16] Yan JH, Pan L, Zhang XM, Sun CX, Cui GH. Lack of efficacy of Tai Chi in improving quality of life in breast cancer survivors: a systematic review and meta-analysis. Asian Pacific Journal of Cancer Prevention Apjcp. Vol. 15, No. 8, 2014, pp. 3715-3720.

[17] Lee MS, Choi TY, Ernst E. Tai chi for breast cancer patients: a systematic review. Breast Cancer Research \& Treatment. Vol. 120, No. 2, 2010, pp. 309-316.

[18] Lee MS, Pittler MH, Ernst E. Is Tai Chi an effective adjunct in cancer care? A systematic review of controlled clinical trials. Support Care Cancer. Vol. 15, No. 6, 2007, pp. 597-601.
[19] Probst P, Grummich K, Heger P, Zaschke S, Knebel P, Ulrich A, Büchler MW, Diener MK. Blinding in randomized controlled trials in general and abdominal surgery: protocol for a systematic review and empirical study. Syst Rev. 2016, 24; 5: 48 .

[20] Liu L, Petrich S, McLaren B, Kelly L, Baxter GD.An integrative Tai Chi program for patients with breast cancer undergoing cancer therapy: study protocol for a randomized controlled feasibility study. J Integr Med. Vol. 16, No. 2, 2018, pp. 99-105.

[21] Pan Y, Yang K, Shi X, Liang H, Zhang F, Lv Q. Tai chi chuan exercise for patients with breast cancer: a systematic review and meta-analysis. Evid Based Complement Alternat Med. 2015, 2015: 535237.

[22] Créquit P, Trinquart L, Yavchitz A, Ravaud P. Wasted research when systematic reviews fail to provide a complete and up-to-date evidence synthesis: the example of lung cancer.BMC Med. 2016 Jan 20; 14: 8.

[23] Yan JH, Pan L, Zhang XM, Sun CX, Cui GH. Lack of efficacy of Tai Chi in improving quality of life in breast cancer survivors: a systematic review and meta-analysis. Asian Pac J Cancer Prev. Vol. 15, No. 8, 2014, pp. 3715-20.

[24] Zeng Y, Luo T, Xie H, Huang M, Cheng AS. Health benefits of qigong or tai chi for cancer patients: a systematic review and meta-analyses. Complement Ther Med. Vol. 22, No. 1, 2014, pp. 173-86. 